Case Report

\title{
Takotsubo Cardiomyopathy in a Patient with Undiscovered Sigmoid Colon Cancer
}

\author{
Huang Po-Yen and Ku Po-Ming \\ Department of Cardiology, Chi-Mei Medical Center, Liuoying, Taiwan \\ Correspondence should be addressed to Huang Po-Yen; boyen0828@gmail.com
}

Received 1 October 2016; Revised 28 November 2016; Accepted 19 December 2016; Published 9 March 2017

Academic Editor: Assad Movahed

Copyright (C) 2017 Huang Po-Yen and Ku Po-Ming. This is an open access article distributed under the Creative Commons Attribution License, which permits unrestricted use, distribution, and reproduction in any medium, provided the original work is properly cited.

\begin{abstract}
Takotsubo cardiomyopathy (TTC) is a stress-related cardiomyopathy that is characterized by reversible left systolic dysfunction, which appears to be precipitated by sudden emotional or physical stress in the absence of myocardial infarction. Here we present a rare case that clinically presented with intermittent abdominal pain, initially impressed as non-ST elevation myocardial infarction and congestive heart failure but with a normal coronary angiogram. Her symptoms relieved spontaneously without returning. Sigmoid colon cancer was diagnosed via colonoscopy later due to persistent abdominal discomfort. In the absence of detectable emotional or physical stress factors, the newly diagnosed sigmoid colon cancer was the only possible trigger factor of TTC. We offer this case as a reminder that cancer should be considered in the differential diagnosis of patients presenting with the etiology of TTC.
\end{abstract}

\section{Introduction}

Takotsubo cardiomyopathy (TTC) is characterized by reversible left systolic dysfunction, which appears to be precipitated by sudden emotional or physical stress in the absence of myocardial infarction. It is also known as "stress-related cardiomyopathy," "left ventricular apical ballooning," "ampulla cardiomyopathy," and "broken heart syndrome." The most commonly used criteria were proposed by the Mayo Clinic and were revised in 2008 [1]. Patients must satisfy all criteria, including the following: (1) transient hypokinesis, akinesis, or dyskinesis in the left ventricular mid-segments with or without apical involvement; regional wall motion abnormalities that extend beyond a single epicardial vascular distribution; and, frequently but not always, a stressful trigger; (2) the absence of obstructive coronary disease or angiographic evidence of acute plaque rupture; (3) new electrocardiogram (ECG) abnormalities or modest elevation in cardiac troponin; and (4) the absence of pheochromocytoma or myocarditis. Although cancer patients have many comorbidities, TTC is rare among cancer patients. On the other hand, cancer may play a role as a trigger of TTC.

\section{Case Report}

A 58-year-old Taiwanese woman was brought to the emergency department (ED) due to intermittent epigastric pain that had lasted for weeks but became exacerbated in the previous few hours. Intermittent lower abdominal pain during defecation with tenesmus was also noted for about 1 month. Other relative symptoms included mild dyspnea on exertion, diaphoresis, hiccupping, and vomiting, which became worse in recent days. She had no past medical history of hypertension or diabetes mellitus. She also had neither known family history nor a history of smoking or drug abuse. There was no postural nocturnal dyspnea, palpitation, or radiation pain. She had no body weight changes in the past 6 months.

A physical examination at the ED revealed a regular heart rate at 75 beats per min, a blood pressure of $154 / 101 \mathrm{mmHg}$, a grade 2 systolic heart murmur at the apex, and clear breathing sounds on auscultation, mild tenderness at the epigastric area, and no edema of the limbs. An initial ECG showed a premature atrial contraction and insignificant ST-T changes (Figure 1(a)). Initial laboratory tests showed mild leukocytosis (WBC: $10000 / \mu \mathrm{L}$, segment: $84.1 \%$ ), Hb $14.4 \mathrm{~g} / \mathrm{dL}$, 


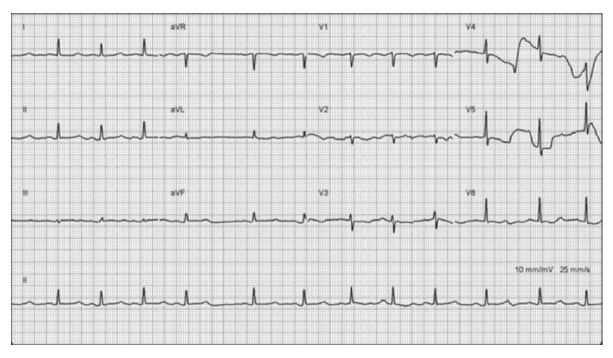

(a)

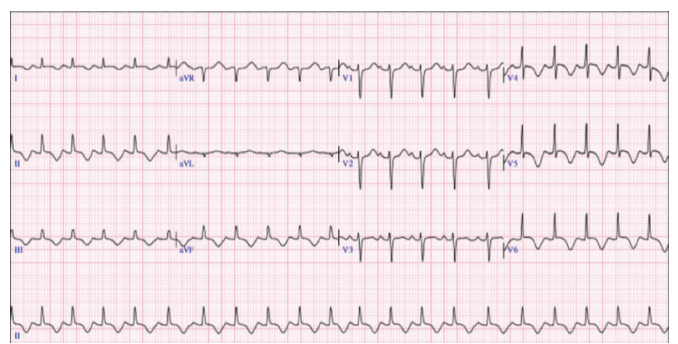

(b)

FIGURE 1: (a) Initial electrocardiogram (ECG) of the patient at the emergency department (ED). (b) Follow-up ECG at the ward 24 hours later.

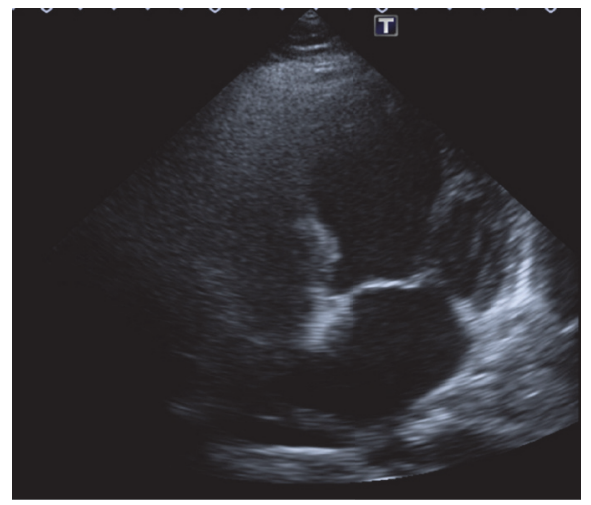

(a)

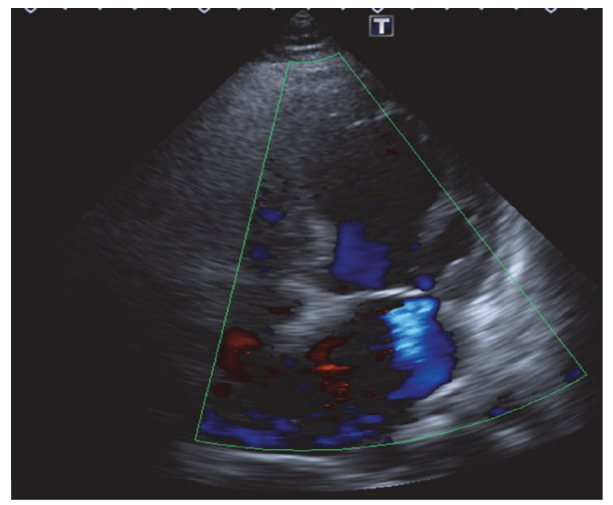

(b)

FIGURE 2: (a) Left ventricle apical hypokinesis during the systolic phase. (b) Mild to moderate mitral valve regurgitation was noted.

platelet count $163000 / \mu \mathrm{L}$, glutamic pyruvic transaminase (GPT) $22 \mathrm{IU} / \mathrm{L}$, creatinine $1.0 \mathrm{mg} / \mathrm{dL}$, troponin $\mathrm{I} 0.06 \mathrm{ng} / \mathrm{mL}$, and creatinine kinase $\mathrm{MB} 0.8 / \mathrm{ng} / \mathrm{Ml}$. After fluid hydration, the patient was admitted to the gastrointestinal ward for suspected peptic ulcer disease. At the ward, a pan-endoscopy showed only mild erythematous gastritis, and the procedure went smoothly without obvious discomforts. However, her epigastric pain seemed stronger and she developed palpitation, diaphoresis, and vomiting at the ward on the following day. Due to the exacerbated symptoms at the ward, a second ECG was performed, which showed new diffused $\mathrm{T}$ wave inversion over leads II, III, aVF, and V4-6 (Figure 1(b)). A follow-up laboratory test showed elevated cardiac enzymes: troponin I $2.26 \mathrm{ng} / \mathrm{mL}$ (normal $<0.05 ; 0.06 \mathrm{ng} / \mathrm{mL}$ at ED) and creatinine kinase $\mathrm{MB} 10.3 \mathrm{ng} / \mathrm{mL}$ (normal < 3.6; $0.8 \mathrm{ng} / \mathrm{mL}$ at ED). A cardiovascular section was then assessed. A transthoracic echocardiogram revealed mild to moderate mitral regurgitation and anterior-apical wall hypokinesis with ejection fraction of $41 \%$ (Figures 2 (a) and 2(b)). The patient was then transferred to the intensive care unit, and cardiac catheterization was arranged for suspected non-ST elevation myocardial infarction. A coronary angiography showed patent coronary arteries (Figures 3(a) and 3(b)) and a left ventriculography showed apical ballooning of the left ventricle, compatible with TTC (Figure 3(c)). The symptoms improved after several days' treatment with diuretics (furosemide, $20 \mathrm{mg}$ twice per day) and propranolol (10 mg, twice per day).

In searching for her triggering factors, there were no detectable emotional or physical factors, including the results of an endocrine survey. The patient was then discharged a few days later with improved symptoms. At OPD, a colonoscopy was further arranged due to intermittent lower abdominal pain during defecation, which accidentally revealed a circumscribed mass with near total obstruction of the sigmoid colon about $20 \mathrm{~cm}$ from the anal verge (Figure 4). Her tumor markers were within the normal range. The patient was then referred to a surgeon for laparoscopic radical sigmoidectomy, and the biopsy showed adenocarcinoma of the sigmoid colon (cT3N0M0). A follow-up ECG 3 months later revealed an improved ejection fraction of $70 \%$ without regional wall motion abnormalities or significant mitral regurgitation. No recurrent angina or dyspnea was present, and the patient is currently being followed up at the oncology OPD.

\section{Discussion}

It is hard to distinguish TTC from acute coronary syndrome in the early clinical stages. Nearly $90 \%$ of reported cases of stress-induced cardiomyopathy occurred in postmenopausal women [2]. TTC may account for up to $2 \%$ of overall acute coronary syndrome cases and has a low mortality rate 


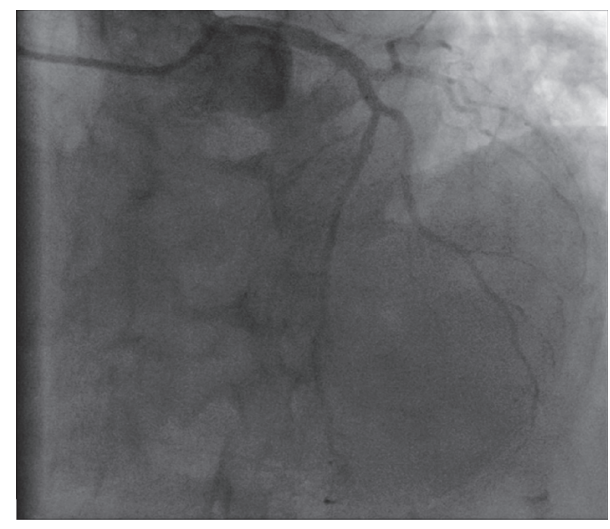

(a)

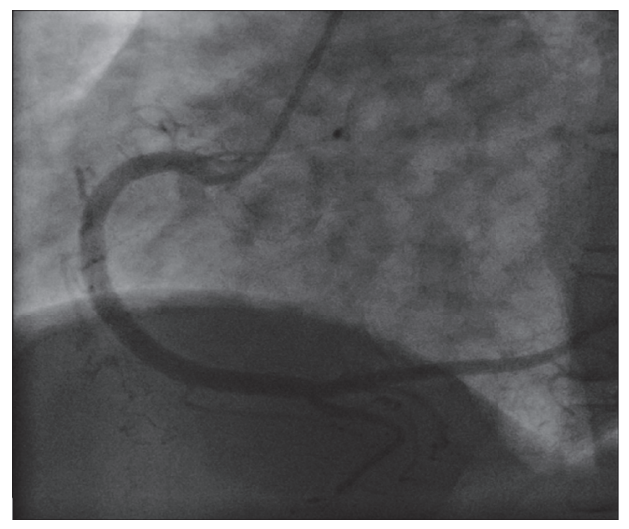

(b)

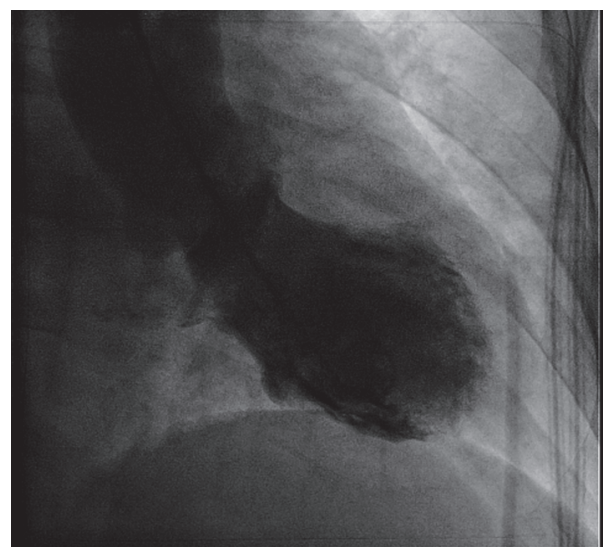

(c)

Figure 3: (a), (b) Patent left and right coronary arteries and (c) typical apical ballooning of the left ventricle at the systolic phase.

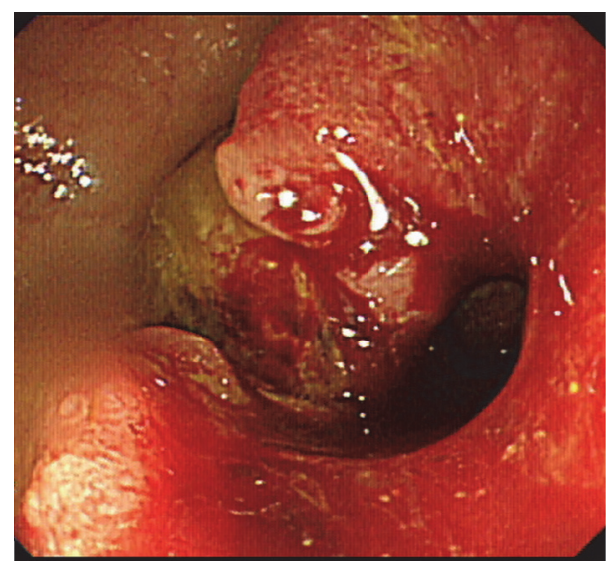

FIgURE 4: A circumscribed mass with near total obstruction of the sigmoid colon.

of less than $2 \%$ and a recovery rate greater than $95 \%$ [3, 4]. The etiology of TTC is thought to be related to stress and catecholamine-induced myocardial injury with elevated norepinephrine levels noted in many patients [5]. About $44 \%$ of the stressful events that initiate TTC are emotional in nature, and $36 \%$ are physical [6].
In the case described here, the persistent epigastric and lower abdominal symptoms lasted for weeks and eventually developed into dyspnea after left ventricular dysfunction proceeded. It is possible that the TTC was induced by panendoscopy [7], but the procedure went smoothly and did not induce any intensive pain or vomiting. Atypical angina and dyspnea already occurred before the invasive examination. Procedure-related stress cardiomyopathy cannot be excluded but the probability is low. The patient presented with LV apical hypokinesis both on ECG and ventriculography with transient mitral regurgitation, which has been reported before [8-10].

Among TTC cases overall, cancer has been rarely reported. Among the cancer-related TTC cases, lymphoproliferative disorder (30\%), gastrointestinal cancer (15\%), and lung cancer (12.5\%) were among the most common types observed [11]. Among these patients, perisurgical procedures (such as abdominal surgery or stem cell transplant) were the most common trigger factors (37.5\%), followed by chemotherapeutic agents use (13.16\%). Emotional stress related to both cancer diagnosis and treatment was also likely a contributor, as patients diagnosed with cancer have been shown to have significantly higher rates of anxiety and depression [12]. Other stressors include changes in their home and family life and fear of disease or death, and those 
with chronic psychological stress are at risk of developing TTC, which is then triggered by an acute stressor [13]. There have been rare case reports of TTC noted before a diagnosed cancer history. Burgdorf and colleagues have reported a $23.6 \%$ incidence of cancer in a cohort of 191 patients with TTC [14], and about 14\% new diagnosed malignancy rate in TTC group after a 3-year follow-up (the rate in the control group was zero). This suggests that malignancy may contribute to the pathophysiology of TTC. In some hypotheses, the effects of catecholamines on myocardial function may be induced by a switch in the $\beta 2$-adrenergic receptor $(\beta 2$ AR) intracellular signalling from a Gs to Gi protein [15], which further increases the level of inflammatory mediators. Inflammation may lead to activation of p38 mitogenactivated protein kinase, a downstream effector of the $\beta 2$ AR signalling pathway that has a negative inotropic effect [16-18]. The relationship between malignancy, inflammation, and myocardial damage still requires further investigation. In conclusion, malignancy screening should be considered if available in patients with unknown cause of TTC.

\section{Consent}

Written informed consent was not available because of change of residence but oral agreement from the patient was obtained.

\section{Conflicts of Interest}

The authors declare that they have no conflicts of interests.

\section{References}

[1] Y. J. Akashi, D. S. Goldstein, G. Barbara, and T. Ueyama, "Takotsubo cardiomyopathy: a new form of acute, reversible heart failure," Circulation, vol. 118, no. 25, pp. 2754-2762, 2008.

[2] M. Gianni, F. Dentali, A. M. Grandi, G. Sumner, R. Hiralal, and E. Lonn, "Apical ballooning syndrome or takotsubo cardiomyopathy: a systematic review," European Heart Journal, vol. 27, no. 13, pp. 1523-1529, 2006.

[3] M. Previtali, A. Repetto, S. Panigada, R. Camporotondo, and L. Tavazzi, "Left ventricular apical ballooning syndrome: prevalence, clinical characteristics and pathogenetic mechanisms in a European population," International Journal of Cardiology, vol. 134, no. 1, pp. 91-96, 2009.

[4] T. M. Pilgrim and T. R. Wyss, "Takotsubo cardiomyopathy or transient left ventricular apical ballooning syndrome: a systematic review," International Journal of Cardiology, vol. 124, no. 3, pp. 283-292, 2008.

[5] T. Ueyama, E. Senba, K. Kasamatsu et al., "Molecular mechanism of emotional stress-induced and catecholamine-induced heart attack," Journal of Cardiovascular Pharmacology, vol. 41, supplement 1, pp. S115-S118, 2003.

[6] J.-W. Lee, H.-K. Lim, J.-Y. Kim, and S. Ryoo, "Stress-induced cardiomyopathy: clinical observations," in CardiomyopathiesFrom Basic Research to Clinical Management, J. Veselka, Ed., chapter 29, pp. 978-953, InTech, Rijeka, Croatia, 2012.

[7] A. R. Lyon, E. Bossone, B. Schneider et al., "Current state of knowledge on Takotsubo syndrome: a Position Statement from the Taskforce on Takotsubo Syndrome of the Heart Failure
Association of the European Society of Cardiology," European Journal of Heart Failure, vol. 18, no. 1, pp. 8-27, 2016.

[8] R. Citro, F. Rigo, Q. Ciampi et al., "Echocardiographic assessment of regional left ventricular wall motion abnormalities in patients with tako-tsubo cardiomyopathy: comparison with anterior myocardial infarction," European Journal of Echocardiography, vol. 12, no. 7, pp. 542-549, 2011.

[9] R. Citro, F. Rigo, A. D’Andrea et al., "Echocardiographic correlates of acute heart failure, cardiogenic shock, and in-hospital mortality in tako-tsubo cardiomyopathy," JACC: Cardiovascular Imaging, vol. 7, no. 2, pp. 119-129, 2014.

[10] F. Pelliccia, G. Parodi, C. Greco et al., "Comorbidities frequency in Takotsubo syndrome: an international collaborative systematic review including 1109 patients," The American Journal of Medicine, vol. 128, no. 6, pp. 654.e11-654.e19, 2015.

[11] P. Vejpongsa, J. Banchs, M. Reyes et al., “Takotsubo cardiomyopathy in cancer patients: triggers, recovery, and resumption of therapy," Journal of the American College of Cardiology, vol. 65, no. 10, p. A927, 2015.

[12] D. Bodurka-Bevers, K. Basen-Engquist, C. L. Carmack et al., "Depression, anxiety, and quality of life in patients with epithelial ovarian cancer," Gynecologic Oncology, vol. 78, no. 3, pp. 302-308, 2000.

[13] I. S. Wittstein, D. R. Thiemann, J. A. C. Lima et al., "Neurohumoral features of myocardial stunning due to sudden emotional stress," New England Journal of Medicine, vol. 352, no. 6, pp. 539548, 2005.

[14] C. Burgdorf, V. Kurowski, H. Bonnemeier, H. Schunkert, and P. W. Radke, "Long-term prognosis of the transient left ventricular dysfunction syndrome (Tako-Tsubo cardiomyopathy): focus on malignancies," European Journal of Heart Failure, vol. 10, no. 10, pp. 1015-1019, 2008.

[15] A. R. Lyon, P. S. C. Rees, S. Prasad, P. A. Poole-Wilson, and S. E. Harding, "Stress (Takotsubo) cardiomyopathy-a novel pathophysiological hypothesis to explain catecholamine-induced acute myocardial stunning," Nature Clinical Practice Cardiovascular Medicine, vol. 5, no. 1, pp. 22-29, 2008.

[16] P. Arabacilar and M. Marber, "The case for inhibiting p38 mitogen-activated protein kinase in heart failure," Frontiers in Pharmacology, vol. 6, article 102, 2015.

[17] M. Girardey, L. Jesel, U. Campia et al., "Impact of malignancies in the early and late time course of takotsubo cardiomyopathy," Circulation Journal, vol. 80, no. 10, pp. 2192-2198, 2016.

[18] S. Land, S. A. Niederer, W. E. Louch et al., "Computational modeling of Takotsubo cardiomyopathy: effect of spatially varying $\beta$-adrenergic stimulation in the rat left ventricle," American Journal of Physiology-Heart and Circulatory Physiology, vol. 307, no. 10, pp. H1487-H1496, 2014. 


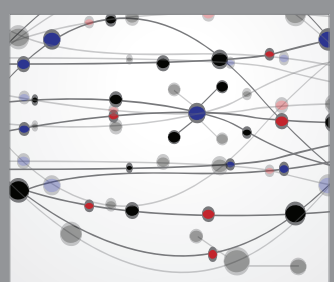

The Scientific World Journal
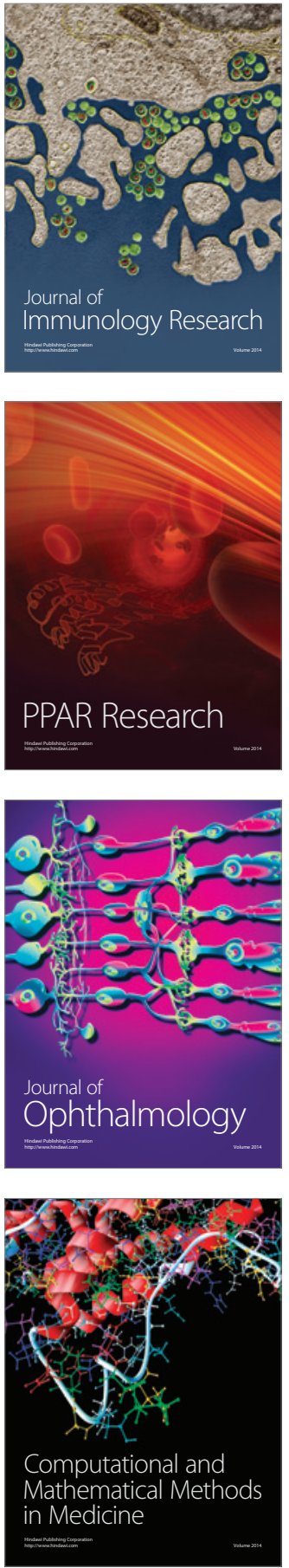

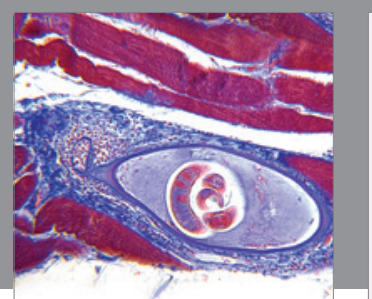

Gastroenterology Research and Practice
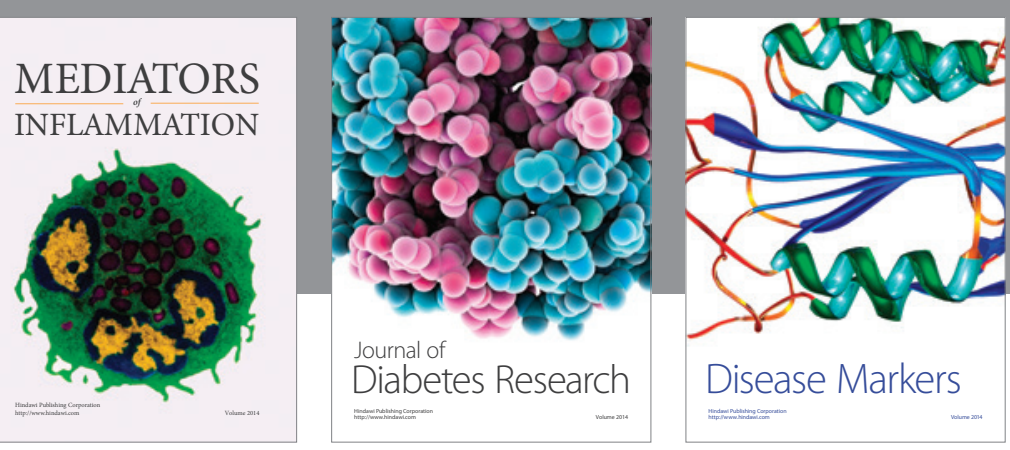

Disease Markers

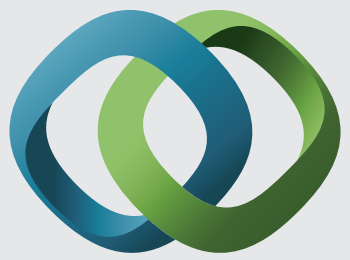

\section{Hindawi}

Submit your manuscripts at

https://www.hindawi.com
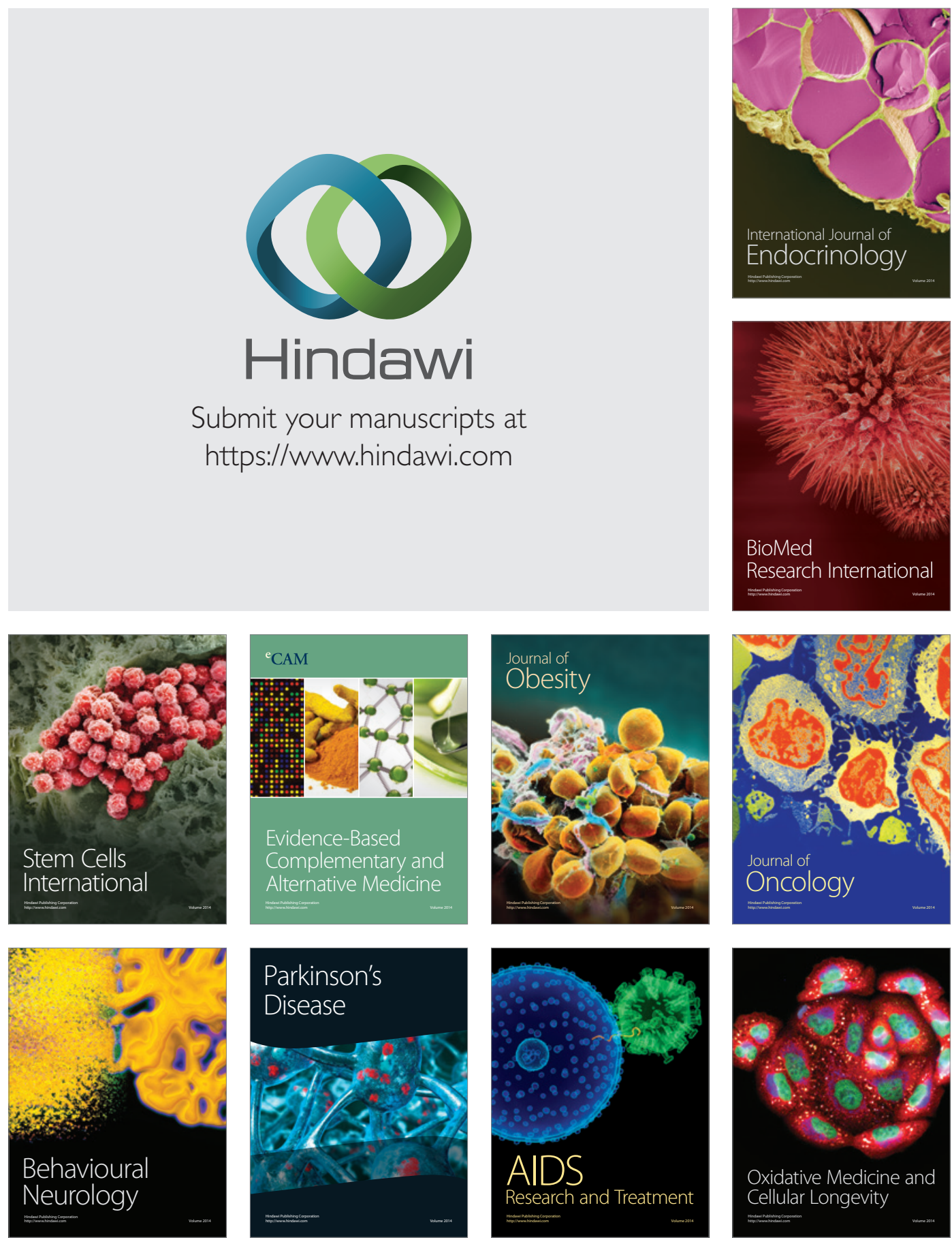